\title{
A network pharmacology and molecular docking study on treatment mechanism of Bacterial Dysentery of Huanglian- Huangqin-Huangbo herb pair
}

qiaoxin xu ( $\nabla$ xqxwenhua99@163.com )

Guangdong Pharmaceutical College: Guangdong Pharmaceutical University

\section{Xiaojia Wang}

Guangdong Panyu District Maternal and Child Health Care Hosptial

\section{Ning Zhong}

Guangdong Pharmaceutical College: Guangdong Pharmaceutical University

\section{Yue Wang}

Guangdong Pharmaceutical College: Guangdong Pharmaceutical University

\section{Zhong Li}

Guangdong Pharmaceutical College: Guangdong Pharmaceutical University

\section{Yanqu Cai}

Guangdong Pharmaceutical College: Guangdong Pharmaceutical University

\section{Research}

Keywords: Coptis chinensis, Scutellaria, Phellodendron, drug pair, bacillary dysentery, network pharmacology, molecular docking, mechanism of action

Posted Date: January 20th, 2021

DOI: https://doi.org/10.21203/rs.3.rs-147874/v1

License: (c) (1) This work is licensed under a Creative Commons Attribution 4.0 International License. Read Full License 


\section{Abstract}

Background: Bacillary dysentery (BD) is one of the most common epidemic infectious diseases. Hundreds of millions of people are infected with BD each year among the world. The patients usually have the following symptoms: abdominal pain, diarrhea, intestinal flora imbalance, etc. Antibiotic are widely used for the treatment in clinical practice. However, due to the overuse of antibiotics, the bacterial resistance is increasingly serious and the medical works are facing with the risk that the antibiotics would lose efficacy. Apart from chemical medicines, traditional Chinese medicines (TCM) are also well accepted for BD treatment, especially in Asian countries. Huanglian-Huangqin-Huangbo herb pair $(\mathrm{HHH})$ is typical and commonly used to treat symptoms such as abdominal pain, diarrhea, and intestinal flora imbalance caused by BD. Also, the HHH has antibacterial, anti-inflammatory, and antidiarrheal effects. In this study, we are committed to ascertain the potential active compounds of $\mathrm{HHH}$ and the onset mechanism for the treatment of $\mathrm{BD}$.

Methods With the help of the Traditional Chinese Medicine Systems Pharmacology Database and Analysis Platform (Traditional Chinese Medicine Systems Pharmacology Database, TCMSP) and PubChem database to search and screen the chemical components and targets of Coptis, Scutellaria, Phellodendron, the gene names were corrected through the Uniprot database, and used the CTD database, TTD database, GeneCards database and DRUGBANK database to obtain BD-related disease targets. The online drawing platform Bioinformatics was used to analyze the "active compound-disease" intersection target, and utilized Cyoscape 3.7.2 software to construct a visualized Chinese medicine-active compound-target network and protein interaction network in order to screen the potential key active compounds and key targets; GO function analysis and KEGG pathway enrichment analysis of the target were carried out through the Metascape database platform, and Cyoscape 3.7.2 software was used to construct a gene-pathway network to screen potential pathways and their mechanism of action. Molecular docking of the key active compounds of the HHH with the key target of BD.

Results A total of 331 potential active compounds were screened for the $\mathrm{HHH}$, among which 87 key active compounds such as quercetin, wogonin, baicalein, $\beta$-sitosterol, isofumarine, and tetrahydroberberine can be selected. Act on BD through 34 potential intersection targets such as IL-6, AKT1, PTGS2, TNF, CASP3, VEGFA, etc. GO gene function analysis yielded a total of 20 biological process (BP) items, 7 cell composition (CC) items, and molecular function (MF) items $(P<0.01)$, mainly involving lipopolysaccharide reaction, reactive oxygen metabolism process, cell factor receptor binding, inorganic substance response, membrane raft, cytokine receptor binding and other biological processes. KEGG pathway enrichment analysis identified 14 signaling pathways $(P<0.01)$, mainly related to cancer signaling pathways, IL-17 signaling pathways and other key pathways. The results of molecular docking $\mathrm{HHH}$ owed that the core active components such as quercetin, $\beta$-sitosterol, wogonin, isofumarole, baicalein and other core active compounds have good binding effects with the core targets of TNF, IL6, PTGS2, and BCL2 (binding energy $<-5 \mathrm{KJ} / \mathrm{mol}$ ).

Conclusion The effect of HHH on the potential key targets of TNF, IL-6, PTGS2 and other potential key targets through quercetin, $\beta$-sitosterol and other potential active compounds to regulate IL-17 and other signaling pathways, thereby exerting therapeutic effects on bacteria. The effect of dysentery is in line with the remarkable characteristics of multi-component, multi-target, and multi-channel effect of Chinese medicine compound.

\section{Background}

Bacillary dysentery, also known as BD, which is an intestinal infectious disease caused by dysentery bacillus (Shigella Castellani) and the features of BD are that it has rapid transmission rate, wide epidemic range, obvious seasonality [1] and people are likely to be infected. Also, BD is internationally recognized as one of most important infectious diseases [2] with its disease incidence and case mortality ranking first in diarrheal diseases worldwide. According to statistical data, there are about 165 million people who get infected with BD each year in the world and about 1.1 million cases die, which is more common in developing countries with poor sanitary conditions such as the Africa [3]. In China, the top five diseases with most morbidity are viral hepatitis, tuberculosis, syphilis, gonorrhea and bacterial and amoebic dysentery , respectively [4].

Loading [MathJax]/jax/output/CommonHTML/jax.js

Page 2/20 
The Shigella is the major pathogen of the onset of BD and there are four common serogroups including Sh.flexneri (group

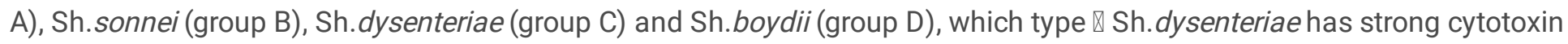
and it could act on the intestinal mucosa to lead to its congestion, edema, ulcers and even necrosis, causing intestinal dysfunction, inducing fever, nausea and vomiting, abdominal pain, diarrhea, tenesmus, purulent bloody stools and other clinical symptoms. What is worse, it could also cause systemic complications, such as electrolyte imbalance, epileptic seizure and hemolytic uremic syndrome[5-6]. Antibiotics are the drugs of choice in the clinical treatment of BD and quinolones (norfloxacin,ofloxacin,ciprofloxacin,etc.),sulfonamides(trimethoprim-sulfamethoxazole, etc.), cephalosporins (ceftriaxone, cefotaxime, etc.), and penicillin (ampicillin, etc.) are commonly used[7] , however, all of them have been proved to serious drug resistance and are gradually facing the risk of becoming invalid and no available drug to choose[8-9].

As a traditional Chinese medicine (TCM) therapy with most characteristics in China, some TCM masterpieces such as Treatise on Febrile Diseases, Puji Fang and so on have records on the treatment of BD. Those treatment could be dialectically classified as: damp-heat dysentery, cold-dampness dysentery, epidemic toxic dysentery, yin deficiency dysentery, aesthetic cold dysentery, and rest dysentery [10]. By searching the database of TCM recipes with the keywords of "dysentery" and "bacillary dysentery", we found that the recipe under the guidance of TCM theory such as Sanhuang Tablets [11] , Huanglian Jiedu Decoction [12] , Shaoyao Decoction [13] , Pulsatilla Decoction [14] , Gegen Qinlian Decoction [15] have a significant effect on the clinical treatment of BD and a variety of dosage form products such as tablets, pills, powders, capsules and injections have been developed [16].

Huanglian-Huangqin-Huangbo herb pair $(\mathrm{HHH})$, consisting of Coptis chinensis Franch. (Huanglian in Chinese), Scutellaria baicalensis Georgi. (Huangqin in Chinese) and Phellodendron amurense Rupr. (Huangbo in Chinese), is commonly used in clearing away internal heat and dampness, purging heat and detoxifying in the TCM clinical practice which could clear the internal heat of upper, middle, lower energizer in human body [17]. Nowadays, there are many research which show that $\mathrm{HHH}$ has an inhibitory effect on pathogenic bacteria such as Shigella, Escherichia coli, Staphylococcus aureus and Salmonella [18-20]. These bacteria are corresponding to the pathogeny of BD. However, the active compounds and potential targets of $\mathrm{HHH}$ in the treatment of $\mathrm{BD}$ have not been found, and the further research on its molecular mechanism is not enough yet.

Network pharmacology is a new discipline which bases on the theory of systems biology. Through analyzing the network of biological systems, selecting specific signaling nodes for multi-target drug molecular design, emphasizing the multipathway regulation of signaling pathways, network pharmacology could reflect the characteristics of "multi-component, multi-target, and multi-pathway" of traditional Chinese herbal compounds [21]. In this study, we predicted the possible targets of BD which are likely to be stimulated by different active compounds in $\mathrm{HHH}$. Meanwhile, we researched for potential important ingredients and key targets and explored the overall regulatory mechanism of multiple components, multiple targets, and multiple pathways of $\mathrm{HHH}$ action.

\section{Methods}

\section{Active compounds and targets of $\mathrm{HHH}$}

Through the Traditional Chinese Medicine Systems Pharmacology Database and Analysis Platform (TCMSP,https://tcmspw.com/molecule.php?qn=1925), by searching Huanglian, Huangqin and Huangbo to collect the potential targets of $\mathrm{HHH}$, then screening the compounds using filters with $\mathrm{OB}$ (oral bio-availability) $\geq 30 \%$ and $\mathrm{DL}$ (drug likeness) $\geq 0.18$ to obtain the active compounds database of $\mathrm{HHH}$. Furthermore, by using Pub chem (https://pubchem.ncbi.nlm.nih.gov/) to grasp the molecular formula, CAS number and download the two-dimension structure in SDF format. By utilizing Uniprot database(https://www.uniprot.org/uploadlists/) to obtain the formula gene names in order to build up a database of active compound and potential targets of $\mathrm{HHH}$.

\section{Targets of bacillary dysentery}

Loading [MathJax]/jax/output/CommonHTML/jax.js 
Based on Gene-cards (https://www.genecards.org/), The Comparative Toxicogenomics Database (CTD, http://ctdbase.org/), Therapeutic Target Database (TTD, http://db.idrblab.net/ttd/) and Drug-bank (https://www.drugbank.ca/), search for the keyword "bacillary dysentery", collect the related target information and delete the information which is repetitive in order to construct a BD related target database.

\section{Therapeutic targets of $\mathrm{HHH}$ on BD}

Using the online drawing platform Microbiology Information Platform( http://www.bioinformatics.com.cn/ ), the potential targets of $\mathrm{HHH}$ on the active compounds and BD were compared and analyzed, and their intersections was taken as the potential target of $\mathrm{HHH}$ on the treatment of $\mathrm{BD}$. Then we corrected the target names to official gene symbol.

\section{Network Construction}

We upload the therapeutic target protein of $\mathrm{HHH}$ on BD to the String database (https://string-db.org/ ) in the form of Gene symbol, then we performed the protein-protein interaction (PPI) analysis, the species was set as "Homo sapiens", the data was exported as TSV files, then imported into Cytoscape3.7.2, the node size and color were set to reflect the connectivity degree, the degree of the node indicates the number of connections between this node and other nodes in the network, the network was analyzed for topological attributes with the help of the function "Network analyze" in Cytoscape3.7.2, and the hub targets which degree value are greater than the median were confirmed .

\section{Enrichment analysis}

Gene Ontology (GO) Enrichment and Kyoto Encyclopedia of Genes and Genomes (KEGG) Pathway Enrichment Analysis were carried out using Metascape database (https://metascape.org/gp/index.html) after importing the HHH-BD intersectional targets. Then selected "OFFICEGENESYMBOL" option, chose the "Homosapiens" option of species, selected "Homosapiens" for the background and filtered with $\mathrm{P}<0.01$ as the condition to obtain the biological process of $\mathrm{HHH}$ on the treatment of $\mathrm{BD}$ and related pathways.

\section{Results}

\section{Main active compound of $\mathrm{HHH}$}

In this study, we obtained 331 components of $\mathrm{HHH}$ totally, after screening and filtering we obtained 87 active compounds from the TCMSP database of Huanglian (Coptis chinensis Franch.), Huangqin (Scutellaria baicalensis Georgi) and Huangbo (Phellodendri Chinrnsis Cortex). In result, we collected 14 active compounds which were from Huanglian, 36 in Huangqin, 37 in Huangbo, respectively and listed in table 1.

\section{Tab.1 Part of the active compounds of the $\mathrm{HHH}$}




\begin{tabular}{|c|c|c|c|c|}
\hline \multirow[t]{2}{*}{ traditional Chinese medicine } & \multirow[t]{2}{*}{ Mol ID } & \multirow[t]{2}{*}{ active compound } & OB & \multirow[t]{2}{*}{ DL } \\
\hline & & & प\%口 & \\
\hline \multirow[t]{14}{*}{ Coptis chinensis Franch.『Huanglian $\rrbracket$} & MOL001454 & Berberine & 36.86 & 0.78 \\
\hline & MOL013352 & Obacunone & 43.29 & 0.77 \\
\hline & MOL002894 & Berberrubine & 35.74 & 0.73 \\
\hline & MOL002897 & Epiberberine & 43.09 & 0.78 \\
\hline & MOL002903 & (R)-Canadine & 55.37 & 0.77 \\
\hline & MOL002904 & Berlambine & 36.68 & 0.82 \\
\hline & MOL002907 & Corchoroside A_qt & 104.95 & 0.78 \\
\hline & MOL000622 & Magnograndiolide & 63.71 & 0.19 \\
\hline & MOL000762 & Palmidin A & 35.36 & 0.65 \\
\hline & MOL000785 & Palmatine & 64.6 & 0.65 \\
\hline & MOL000098 & Quercetin & 46.43 & 0.28 \\
\hline & MOL001458 & Coptisine & 30.67 & 0.86 \\
\hline & MOL002668 & Worenine & 45.83 & 0.87 \\
\hline & MOL008647 & Moupinamide & 86.71 & 0.26 \\
\hline \multirow[t]{10}{*}{ Scutellaria baicalensis Georgi囚Huangqin $\rrbracket$} & MOL000073 & ent-Epicatechin & 48.96 & 0.24 \\
\hline & MOL000173 & Wogonin & 30.68 & 0.23 \\
\hline & MOL000358 & beta-sitosterol & 36.91 & 0.75 \\
\hline & MOL000359 & Sitosterol & 36.91 & 0.75 \\
\hline & MOL000449 & Stigmasterol & 43.83 & 0.76 \\
\hline & MOL000525 & Norwogonin & 39.4 & 0.21 \\
\hline & MOL001506 & Supraene & 33.55 & 0.42 \\
\hline & MOL001689 & Acacetin & 34.97 & 0.24 \\
\hline & MOL002714 & Baicalein & 33.52 & 0.21 \\
\hline & MOL002897 & Epiberberine & 43.09 & 0.78 \\
\hline \multirow[t]{8}{*}{ Phellodendron amurense Rupr.『Huangbo® } & MOL001454 & Berberine & 36.86 & 0.78 \\
\hline & MOL001458 & Coptisine & 30.67 & 0.86 \\
\hline & MOL002636 & Kihadalactone A & 34.21 & 0.82 \\
\hline & MOL013352 & Obacunone & 43.29 & 0.77 \\
\hline & MOL002641 & Phellavin_qt & 35.86 & 0.44 \\
\hline & MOL002643 & delta 7-stigmastenol & 37.42 & 0.75 \\
\hline & MOL000790 & Isocorypalmine & 35.77 & 0.59 \\
\hline & MOL002651 & Dehydrotanshinone II A & 43.76 & 0.4 \\
\hline
\end{tabular}




\begin{tabular}{|llll}
\hline MOL002652 & delta7-Dehydrosophoramine & 54.45 & 0.25 \\
\hline MOL002656 & Dihydroniloticin & 36.43 & 0.81 \\
\hline
\end{tabular}

\section{Overlapping analysis}

After statistical analysis, there were 501 related targets corresponding to the active compound of $\mathrm{HHH}$, after removing duplicate items, we collected 228 related targets. Meanwhile we searched the DrugBank, GeneCards, CTD and TTD database with the keyword "bacillary dysentery" and found 0,141, 184, 0 therapeutic targets, respectively. And 313 BD related targets were discovered after removing duplicates. Then we carried overlap analysis between these $228 \mathrm{HHH}$ related targets and BD related targets and 34 frequently affected therapeutic intersection targets for $\mathrm{BD}$ in active compounds of $\mathrm{HHH}$ were discovered. The 34 targets were as follows: AKT1, BAX, BCL2, CASP3, CD40LG, CDKN1A, CRP, CXCL2, CXCL8, CYP1A1, CYP1A2, EGFR, FASLG, GJA1, GSTM2, HMOX1, IFNG, IGFBP3, IL10, IL-1A, IL-13, IL-4, IL-6, MAPK1, MPO, NFKBIA, NOS2, NQ01, PON1, PTGS2, RELA, SOD1, TNF, VEGFA (Fig. 1).

From the "drug-active compound-target" network constructed by HHH active compounds and BD related targets (Fig.2), among them, rhombuses represent medicinal materials, green circles represent the compound of Coptis chinensis, blue circles represent the compound of Scutellaria, red circles represent the compound of Phellodendron, and orange rectangles represent genes. We came to the conclusion that the ingredients in $\mathrm{HHH}$ which of great degree value such as mistletoe bombesin (degree:150), wogonin (degree:45), baicalein (degree:38), $\beta$-sitosterol (degree:37), isobaric monophenol (degree:35), tetrahydroberberine (degree:32), stigmasterol (degree:30), Cavidine (degree:28), aecranine (degree:27), and proopiate (degree:27) may be the main components which exerts drug therapeutic effect.

\section{PPI network of the key target of HHH for the treatment of bacillary dysentery}

34 key targets were imported into the STRING database to obtain the PPI network of component and disease targets. In the PPI network, there were 34 nodes, 303 edges, and the average node degree value was 17.82. The greater the node degree value, the more obvious the biological function it has. Among them(Fig.3), there were 14 targets which degree $>20$, namely IL-6 (degree:29), AKT1 (degree:28), PTGS2 (degree:28), TNF (degree:28), CASP3 (degree:27), VEGFA (degree:27), CXCL8 (degree:26), IL-1B (degree:25), MAPK1 (degree:24), IL-10 (degree:23), IFNG (degree:22), HMOX1 (degree:21), MPO (degree:21), IL-4 (degree:21).

\section{GO enrichment and KEGG enrichment}

Set $P<0.01$ as the cutoff value, GO enrichment analysis and KEGG enrichment analysis of HHH and BD`s shared target genes was performed. GO Biological Processes mainly involves the reaction of lipopolysaccharide, active oxygen metabolism, inorganic substance, cell death regulation and leukocytosis. GO Cellular Components mainly involves membrane raft, components of presynaptic membrane, vesicle cavity, outer membrane of organelle, neuronal cell body and perinuclear area of cytoplasm. GO Molecular Functions mainly involves cytokine receptor binding, protein homologous dimerization activity, heme binding, tumor necrosis factor receptor superfamily binding. KEGG enrichment analysis involved 14 signaling pathways, including cancer signaling pathway, human cytomegalovirus infection signaling pathway, IL-17 signaling pathway and HIF-1 signaling pathway.

\section{Analysis of the "active compound-gene target-pathway" network of $\mathrm{HHH}$}

The "active compound-gene target-pathway" network of HHH was generated using Cytoscape3.7.2 by 87 related active compounds, 34 intersection gene targets and 14 important pathways (Fig. 7).

The network diagram contained 104 nodes and 358 edges, among which 56 red srhombus represented active components (the components unrelated to the pathway have been deleted), 34 green hexagons represented gene targets, 14 orange

Loading [MathJax]/jax/output/CommonHTML/jax.js ${ }^{\text {nd }}$ the edges represented the relationship among compounds, gene targets and 
pathways. According to the degree value, the top 5 Active compounds were screened for molecular verification, namely quercetin (degree: 149), wogonin (degree: 44), scutellarin (degree: 37), $\beta$-sitosterol (degree: 36), and Isocorypalmine (degree: 34).

\section{Molecular docking results}

To further verify the effect, the screened active compounds namely quercetin, $\beta$-sitosterol, scutellarin, wogonin, isocorypalmine and the following potential target proteins: TNF (PDB:1JH5), IL-6 (PDB:4O9H) and PTGS2 (PDB:5KIR) were used for molecular docking. The results (Tab. 2) showed that the binding energy of the target protein and the corresponding active component were less than $-5 \mathrm{~kJ} / \mathrm{mol}$, indicating that the target proteins have good binding property. Three proteins were predicted successfully to be the targets in treatment of $B D$, quercetin, $\beta$-sitosterol, scutellarin, wogonin, and isocorypalmine. Three targets and the lowest binding energy of five ligand components were used to draw the docking mode diagram of the components and targets by PyMol 2.3.4 (Fig.8). The binding sites of $\beta$-sitosterol-PTGS2 were ASP-133 amino acid residues, $\beta$-sitosterol-TNF exerted effects through ASN-102 and GLN-93 amino acid residues, and the binding sites of Baicalein-PTGS2 were HIS-207 and ASN-382 amino acid residues.

Tab.2 Results of molecular docking between core compounds and core targets

\begin{tabular}{|lllllll|}
\hline Mol ID & compound & $\begin{array}{l}\text { Relative } \\
\text { molecular } \\
\text { mass } \\
(\mathbf{g} / \mathbf{m o l})\end{array}$ & Degree & $\begin{array}{l}\text { Binding energy } \\
\text { with IL- } \\
6 /(\mathbf{k J} / \mathrm{mol})\end{array}$ & $\begin{array}{l}\text { Binding energy with } \\
\text { PTGS2/(kJ/mol) }\end{array}$ & $\begin{array}{l}\text { Binding } \\
\text { energy with } \\
\text { TNF }\end{array}$ \\
\hline MOL000098 & Quercetin & 302.25 & 149 & -5.71 & -7.69 & -5.16 \\
\hline MOL000173 & wogonin & 284.28 & 44 & -5.29 & -7.37 & -5.39 \\
\hline MOL000358 & beta-sitosterol & 414.79 & 36 & -7.27 & -70.36 & -8.55 \\
\hline MOL000790 & Isocorypalmine & 341.44 & 34 & -6.02 & -7.21 & -5.89 \\
\hline MOL002714 & baicalein & 270.25 & 37 & -6 & -7.41 & -5.73 \\
\hline
\end{tabular}

\section{Discussion}

Based on the theory of evidence-based medicine of TCM, the form of compound is often applicated and treated clinical of various diseases, but due to the complexity of Chinese native medicine ingredient, existing methods mainly studied around some index ingredients and pharmacological effects. It cannot show the TCM`s mode of systemic effects, "plural ingredients, plural targets" completely, thus to clarify the network relations of different active compound and effect targets is the key to the development of TCM modernization [22]. Network pharmacology research is based on systems biology theory, which can analyze and predict the interaction between components and targets, so as to more comprehensively explain the multi-pathway regulation effects of different components on signal pathways, it is a powerful method to study the systemic effects of TCM compounds [23]. For further analysis, the TCMSP database provided 331 compounds which obtained from $\mathrm{HHH}$ in the study. According to the principle of $A D M E, O B$ reflects the process of drug absorption, distribution, metabolism and excretion, and $\mathrm{DL}$ reflects the similarity between compounds and known drug molecules. It is generally believed that only drugs with $\mathrm{OB} \geq 30 \%$ and $\mathrm{DL} \geq 0.18$ are worthy of development [24-25].

Therefore, 87 active compounds were further screened based on these two indicators in this study, and including 14 active compounds of Huanglian, 36 active compounds of Huangqin, and 37 active compounds of Huangbo. By analyzed the PPI network we found that, Huanglian sassociatedma $\in$ tar $\geq t \geq \neq$ swerePTGS2, NOS2, AKT1, BAX, BCL2, CASP3, CD40LG, CDKN1A, Huangq $\in$

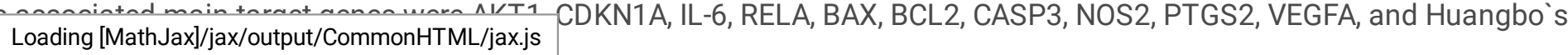


associated main target genes were PTGS2, NOS2, BAX, BCL2, CASP3, CYP1A2, PON1, TNF. In addition, there were 34 targets shared with BD Disease targets, namely IL-6, AKT1, PTGS2, TNF, CASP3, VEGFA and BCL2. For example, Among them, Shigella flora was positively correlated with environmental factors such as IL-6 and TNF-a[26]. PTGS, also known as cyclooxygenase (COX), it divided into two subtypes, namely COX-1 and COX-2. Previous studies have shown that inflammatory response related to PTGS closely [27]. The specifical binds between TNF- $\alpha$ and receptors can induce inflammatory response [28]. And AKT1 is the hub target of PI3K-Akt signaling pathway, which has not only played an important role in the process of promoting cell proliferation and inhibiting apoptosis, but also participated in the process of inflammation. It is essential in intestinal inflammation [29].

Nowadays, it is still unclear of the exact pathogenesis of BD. The main pathogenic process is that the bacteria go through the gastrointestinal barrier to the colon, then invade colonic epithelial cells and they begin to grow and diffuse into adjacent infected cells, cause inflammation, epithelial cell death, ulcers, increase fluid in the colon that produce pus and mucus, etc[30]. It has been reported that Shigella can secrete specific virulence factors, thus disrupting the integrity of the epithelial barrier. In this way, the ability of pathogens to penetrating into colon tissues in the body can be enhanced, it finally induced inflammatory reactions, destroyed colon epithelial cells and produced clinical symptoms[31]. The basic clinical treatment of intestinal diseases is to prevent inflammation from happening and mechanism of action may be realized by regulating Th17/Treg balance and controlling intestinal bacteria [32]. According to KEGG analysis, there are 14 signaling pathways of $\mathrm{HHH}$ `s BD therapy including cancer signaling pathway, human cytomegalovirus infection signaling pathway, IL-17 signaling pathway, HIF-1 signaling pathway, etc. Among them, IL-17 signaling pathway is one of the major signaling pathways involved in regulating inflammatory response. The IL-17 receptor family contain five(5)members, namely IL-17RA, IL-17RB, IL-17RC, IL-17RD, and IL-17RE. The key receptor in inflammatory response is IL-17A among the family. It can stimulates the produce of other pro-inflammatory cytokines such as TNF- $\alpha$ and IL-1 $\beta$, and promote a large number of inflammatory factors produced with cytokines, thus mediating immune inflammatory response [33].

Among the 87 active compounds selected by $\mathrm{HHH}$, such as quercetin, $\beta$-sitosterol, scutellarin, wogonin, and isocorypalmine, scutellarin can effectively inhibit the growth of Shigella bacteria, significantly reduce the content of IL- 6 and TNF-a in serum of CIM mice, and slow down the occurrence of inflammatory reaction [26]. Wogonin has anti-inflammatory effect because it can inhibit the inflammatory process of II-6, TNF-a and other important cytokines [34]. Quercetin can eliminate inflammation in vivo and in vitro by inhibiting inflammatory cytokines such as IL-6 and TNF-a and inflammatory enzymes. Moreover, quercetin has an inhibitory effect on various bacteria such as Escherichia coli and Salmonella, especially its inhibitory effect on gram-negative bacteria is stronger than gram-positive bacteria [35-36]. The $\beta$-sitosterol play an anti-inflammatory role by significantly inhibit the expression of IL-6 and COX-2[37]. Berberine has an obvious inhibitory effect on dysentery bacillus [38]. scutellarin, wogonin, quercetin and other flavonoids and their natural plant extracts can also maintain and repair the integrity of the intestinal mucosa, adjusting the composition of the intestinal microflora. For example, inhibiting the harmful bacteria such as e.coli while promoting the growth of beneficial bacteria such as Bifidobacterium, Bacteroidetes, which regulates intestinal immunity, reduce proinflammatory factor, reduce inflammatory bowel disease caused by a variety of intestinal microecological imbalance[39]. By PPI network, we further screening core active compounds of quercetin, $\beta$ sitosterol, scutellarin, wogonin, isocorypalmine, and core target gene TNF, IL-6 and PTGS2. By using molecular docking, obtained the core binding energy of active compounds and core target gene are less than $-5 \mathrm{KJ} / \mathrm{mol}$, and these data were further proved the accuracy and reliability of the results in this study.

We also found some potential active compounds of $\mathrm{HHH}$, such as obacunone, epiberberine, berberrubine,cantharidin, salviarin, acacetin. Currently, no correlation between these compounds and bacillary dysentery has been reported. However, they play a more important role in other diseases. For example, obacunone can inhibit VSMC proliferation, reduce intimal hyperplasia, and inhibit the occurrence of venous bridge restenosis by down-regulating the phosphorylation level of Akt [40], epiberberine has hypoglycemic effect [41], cantharidin has anti-tumor effect [42], and acacetin can protect the nerve function of rats HAD acute cerebral infarction [43]. In addition, some potential targets were also found, such as EGFR, IFNG, IL-10,

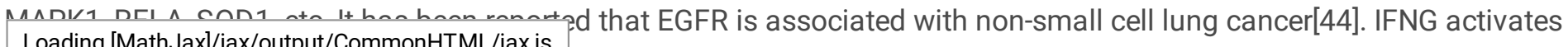
Loading [MathJax]/jax/output/CommonHTML/jax.js 
macrophages, neutrophils, endothelial cells, platelets, as well as complement systems and coagulation systems, and then inducing the release of bioactive substances such as tumor necrosis factor a prostaglandin and kinin, and is widely recognized as a regulator of tumor immune surveillance and tumorigenesis[45]. MAPK1 plays an important role in the pathological process of preeclampsia [46]. RELA is essential for maintaining liver homeostasis against TNF-a driven immunotoxicity [47]. Therefore, the development of potentially active compounds as a new drug for the treatment of BD and the elucidated target gene as a new mechanism of action for the treatment of BD has very important research significance and innovation, which will be carried out in the next work plan.

\section{Conclusion}

Sum up, this study found that except for compound known to work on BD in $\mathrm{HHH}$, such as quercetin, scutellarin, $\beta$-sitosterol, Berberine, there may still exist in other compound more involved in BD compounds of internal like obacunone, epiberberine, Coptisine hydrochloride, cantharidin, salviarin, acacetin. The specific effects of these compounds will need to be confirmed by further experimental studies at the cellular and animal levels.

In addition, important target genes reported in previous studies of $\mathrm{HHH}$, such as IL-6, AKT1, PTGS2, TNF, CASP3, VEGFA, $\mathrm{BCL2}$, may play an important role in alleviating BD abdominal pain, diarrhea, intestinal flora imbalance and other symptoms.

\section{Abbreviations}

BD:Bacillary dysentery;TCM:traditional Chinese medicines;BP:biological process;

CC:cell composition;MF:molecular function;PPI:protein-protein interaction;

GO:Gene Ontology;KEGG:Kyoto Encyclopedia of Genes and Genomes;

HHH:Huanglian-Huangqin-Huangbo;TSCMP:Traditional Chinese Medicine Systems Pharmacology Database; TTD:

Therapeutic target database;CTD:Comparative Toxicogenomics Database;

\section{Declarations}

\section{Acknowledgements}

Not applicable.

\section{Authors' contributions}

YC conceived the research theme and supervised the implementation and Revised final draft. QX and XW designed the method and performed the experiments and wrote the manuscript. NZ and YW revised the language. ZL provided guidance. All authors read and approved the final manuscript.

\section{Funding}

This work was supported by the National Natural Science Foundation of China (Grant No. 2017KSYS002, No. 81373981), the Guangdong Medical Science and Technology Research Fund (Grant No. A2020368).

\section{Availability of data and materials}

The datasets used and/or analyzed in the current study are available from

the corresponding author on request.

Loading [MathJax]/jax/output/CommonHTML/jax.js 
Not applicable.

\section{Consent for publication}

Not applicable.

\section{Competing interests}

The authors declare that they have no competing interests.

\section{References}

1. Qi XQ, Wang Y, Disease Prevention and Control Bureau of the Ministry of Health, etc. Handbook of Dysentery Prevention and Control. Beijing: People's Medical Publishing House.2006.

2. Osorio JJ, Román AR, Torrecisneros J. Spectrum and risk factors of invasive fungal infection. Enferm Infec Micr Cl. 2007;25(7): 467.

3. Sarma A, Singh SL. Molecular epidemiological characteristics of Shigella spp. isolated from river Narmada during 20052006. J Environ Health. 2009;71(6): 61-66.

4. General situation of national legal infectious diseases in June 2020. Chinese Journal of Viral Diseases. 2020;10 (5): 349.

5. WS287-2008 Diagnostic criteria for bacterial and amoebic dysentery.

6. Jin D. Epidemiological analysis of bacillary dysentery in Xuzhou from 2010 to 2014. Journal of Preventive Medicine Information. 2017;33(2): 187-190.

7. Guo SP. Comparative study on drug treatment of bacillary dysentery. Enterprise Herald. 2014;(17): 192-193.

8. Puzari M, Sharma M, Chetia P. Emergence of antibiotic resistant Shigella species: A matter of concern. Journal of Infection and Public Health. 2018;11(4): 451-454.

9. Wang H, Sun N, Yang W. Research progress in Shigella resistance to quinolones. Agricultural Science Research. 2020;41(3): 51-54.

10. Chinese Society of Traditional Chinese Medicine. Guidelines for the clinical diagnosis and treatment of bacillary dysentery in TCM internal medicine. Beijing Traditional Chinese Medicine. 2020;39 (6): 521-525.

11. Yuan LG, Liao GS. Analysis of curative effect of traditional Chinese medicine Sanhuang Tablets combined with Andrographis paniculata on bacillary dysentery. Journal of Yichun Medical College. 2001;(2): 201.

12. Wang JG, Hua MY. Modified Huanglian Jiedu Decoction in the treatment of 52 cases of common acute bacillary dysentery. Journal of Practical Traditional Chinese Medicine. 2005;21 (12): 727.

13. Li JQ. Report of 9 cases of acute bacillary dysentery treated with Shaoyao Decoction. Community Medicine Journal. 2007; (16): 33-34.

14. Wang L, Tao XB, Ouyang Jing, Yang Zhongping. Study on antibacterial effect of Baitouweng decoction on Shigella in vitro. Information on Traditional Chinese Medicine. 2020;37 (5): 49-53.

15. Qu L, Wang H, Chen CM, Li ZG. Comparative observation of Gegen Qinlian Decoction with traditional Chinese medicine decoction granules and traditional decoction pieces in the treatment of bacillary dysentery. Guangming Traditional Chinese Medicine. 2012;27 (2): 265-266.

16. Du Y, Gong LB, Qiu J. Reasonable application of Chinese patent medicine for bacillary dysentery. People's Military Medical. 2018;61 (11): 1065-1066, 1079. 
17. Teng L, Chai YQ, Xin YJ, Yang Y, Wei CY. Based on "Changsha Yaojie" to analyze the clinical effects of Scutellaria baicalensis Georgi, Coptis and Phellodendron amurense. Journal of Shanghai University of Traditional Chinese Medicine. 2018;32 (2) : 7-10.

18. Yin LL. The pharmacological effects of Coptis and modern research progress. Clinical Research of Traditional Chinese Medicine. 2016;8 (28): 144-145.

19. Xu YT. Research progress on the chemical constituents and modern pharmacological effects of Scutellaria baicalensis Georgi. Guangming Traditional Chinese Medicine. 2010;25 (3): 544-545.

20. Hu JQ, Hu X. Modern research on chemical constituents and pharmacological effects of Phellodendron amurense. Contemporary Medicine. 2009;15 (7): 139-141.

21. Yuan H, Ma Q, Cui H, et al. How Can Synergism of Traditional Medicines Benefit from Network Pharmacology?. Molecules. 2017;22 (7):1135.

22. Li S, Zhang B. Traditional Chinese medicine network pharmacology: theory, methodology and application. Chin J Nat Med. 2013;11 (2):110-20.

23. Wang L, Li Z, Shao Q, Li X, Ai N, Zhao X, et al. Dissecting Active compounds of Chinese medicine by content-weighted ingredient-target network. MolBioSyst. 2014;10 (7):1905-11.

24. Dong J, Wang NN, Yao ZJ, Zhang L, Cheng Y, Ouyang D, et al. ADMETlab: a platform for systematic ADMET evaluation based on a comprehensively collected ADMET database. J Cheminform. 2018;10 (1):29.

25. Tian S, Li Y, Wang J, Zhang J, Hou T. ADME evaluation in drug discovery. 9. Prediction of oral bioavailability in humans based on molecular properties and structural fingerprints. Mol Pharm. 2011;8 (3): 841-51.

26. Wang R, Wang L, Wei GY, Liu NN, Zhang L, Wang SM, et al. The effect and mechanism of baicalein in regulating intestinal flora and improving chemotherapy-induced intestinal mucositis in mice. Journal of Pharmaceuticals. $2020 ; 55$ (5): 868-876.

27. Anyona SB, Hengartner NW, Raballah E, Ong'echa JM, Lauve N, Cheng Q, et al. Cyclooxygenase-2 haplotypes influence the longitudinal risk of malaria and severe malarial anemia in Kenyan children from a holoendemic transmission region. J Hum Genet. 2020;65 (2): 99-113.

28. Liu ZG. Molecular mechanism of TNF signaling and beyond. Cell Res. 2005;15(1):24-7.

29. Cheng HJ, Lv XP. Research progress on the relationship between PI3K-AKT signal transduction pathway and inflammatory bowel disease. Guangdong Medicine. 2014; 35 (2): 297-299.

30. Su HP, Wu JL, Yan QC. The pathogenesis of Shigella causing bacillary dysentery. Asian Journal of Clinical Medicine. 2020;3 (1): 76-78.

31. Ferrari ML, Malardé V, Grassart A, Salavessa L, Nigro G, Descorps-Declere S, et al. Sigella promotes major alteration of gut epithelial physiology and tissue invasion by Sutting off host intracellular transport. Proc Natl Acad Sci US A . 2019;116 (27): 13582-13591.

32. Liang S, Deng X, Lei L, Zheng Y, Ai J, Chen L,et al. The Comparative Study of the Therapeutic Effects and Mechanism of Baicalin, Baicalein, and Their Combination on Ulcerative Colitis Rat. Front Pharmacol. 2019;10:1466.

33. Du XB, Gao C, Yang BB, Wang DD, Geng L, Wang XP, et al. Research progress on the relationship between interleukin 17A and inflammatory bowel disease. Journal of Gastroenterology and Hepatology. 2019;28 (5): 573-576.

34. Zhu SL, Huang HL. Research progress on anti-inflammatory mechanism of flavonoids of Scutellaria baicalensis Georgi . Journal of Jiangxi University of Traditional Chinese Medicine. 2010;22 (3): 97-100.

35. Yang Y, Wang YY, Jiang QC. Research progress on the pharmacological effects of quercetin. Special Economic Animals and Plants. 2020;23 (5): 24-28.

36. Liu SW, Liu JY. Research progress on the pharmacological effects of quercetin. Chinese Journal of Lung Diseases (Electronic Edition). 2020;13(1): 104-106. 
37. Feng SM, Ning K, Shao P, Ren GP, Sun PL, Luo ZS. Study on the therapeutic effects of $\beta$-sitosterol and stigmasterol on acute colitis in mice. Journal of the Chinese Cereals and Oils Association. 2018;33 (12): 80-86, 94.

38. Lin Y, Zhang HJ, Han YX, Jiang JD. Inhibitory effect of berberine on intestinal bacteria. China Medical Biotechnology. 2018;13 (4): 335-338.

39. Li M, Lu H, Xu ZJ. Research progress on flavonoid natural products regulating intestinal microbes and improving inflammatory bowel disease. Journal of Henan University of Technology (Natural Science Edition). 2020;41 (3): 118 -129 .

40. Li DY, Chen WJ, SJ, Li SK. The inhibitory effect of corvone on intimal hyperplasia after autologous vein graft graft in rats and its mechanism . Guangxi Medicine. 2018;40 (7): 808-811.

41. Cui XL, Wang DZ, Zou ZY, Hu XR, Li XG. Study on hypoglycemic effect of epiberberine. Asia-Pacific Traditional Medicine. 2015;11 (13): 12-13.

42. Li X, Li SS, Pang JL, Huang FH, Guo B, Liu H. Alkali preparation can significantly enhance the anti-tumor effect of Cantharidum. Journal of Southern Medical University. 2020;40 (9): 1332-

43. Zheng JJ, Yang XM, Mao LQ. Based on TGF- $\beta 1 / S m a d 3$ signaling pathway to explore the protective effect of acacetin on the neurological function of rats with acute cerebral infarction. Chinese Pharmacist. 2020;23 (7): 1350-1354.

44. Zheng J, Xie GY, Li J, Luo JD, Wen QY, Fan SQ. The clinical significance of EGFR gene mutation in non-small cell lung cancer. Chinese Journal of Oncology. 2014;41 (14): 904-907.

45. Zaidi MR. The Interferon-Gamma Paradox in Cancer. J Interferon Cytokine Res. 2019;39(1):30-38.

46. Wang Y, Cheng K, Zhou W, Liu H, Yang T, Hou P, et al. miR-141-5p regulate ATF2 via effecting MAPK1/ERK2 signaling to promote preeclampsia. Biomed Pharmacother. 2019;115:108953.

47. Kim Y, Allen E, Baird LA, Symer EM, Korkmaz FT, Na E, et al. NF-KB RelA Is Required for Hepatoprotection during Pneumonia and Sepsis. Infect Immun. 2019;87 (8): e00132-19.

\section{Figures}




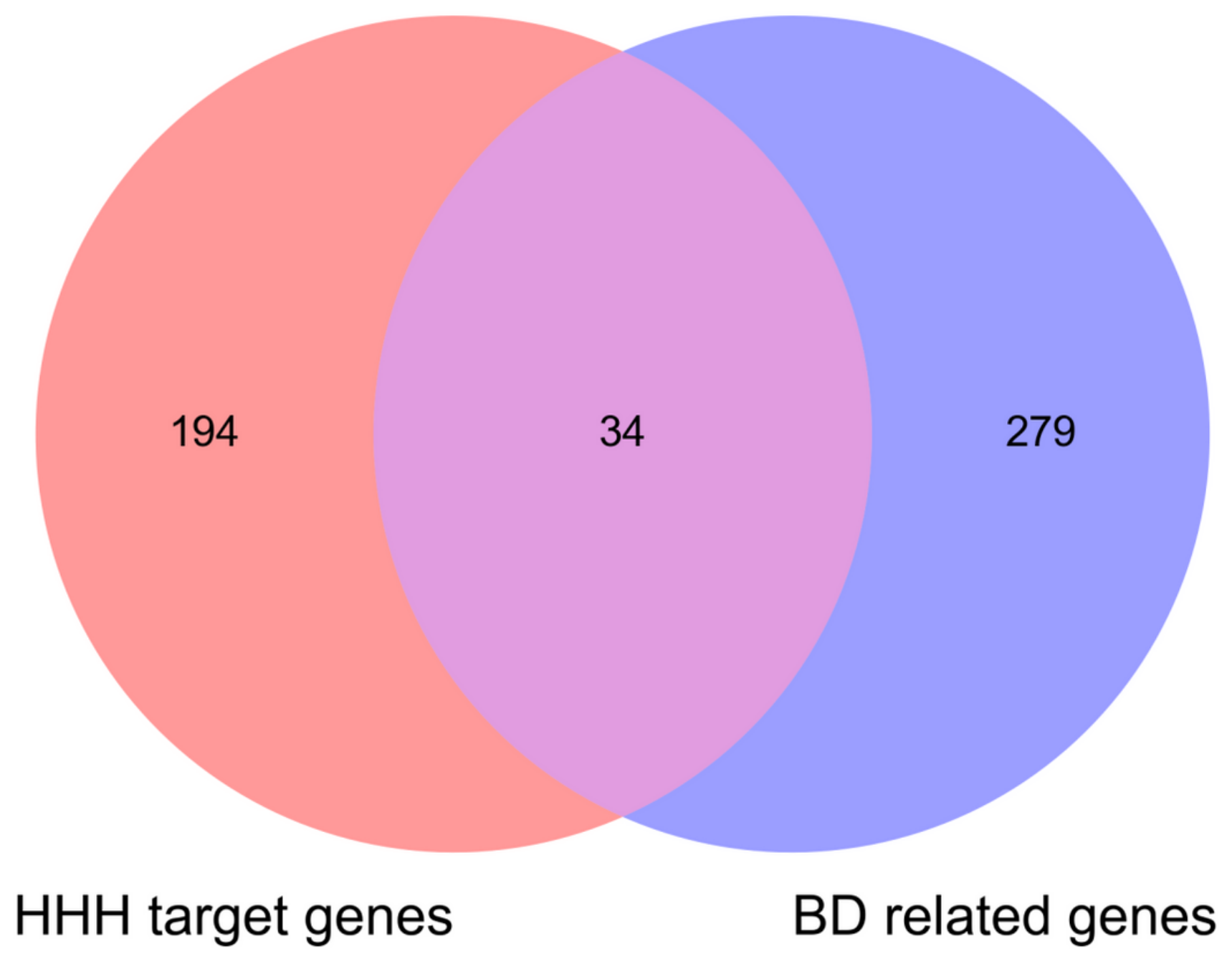

Figure 1

The intersection of bacillary dysentery's disease treatment targets and HI4H's active compounds' targets 


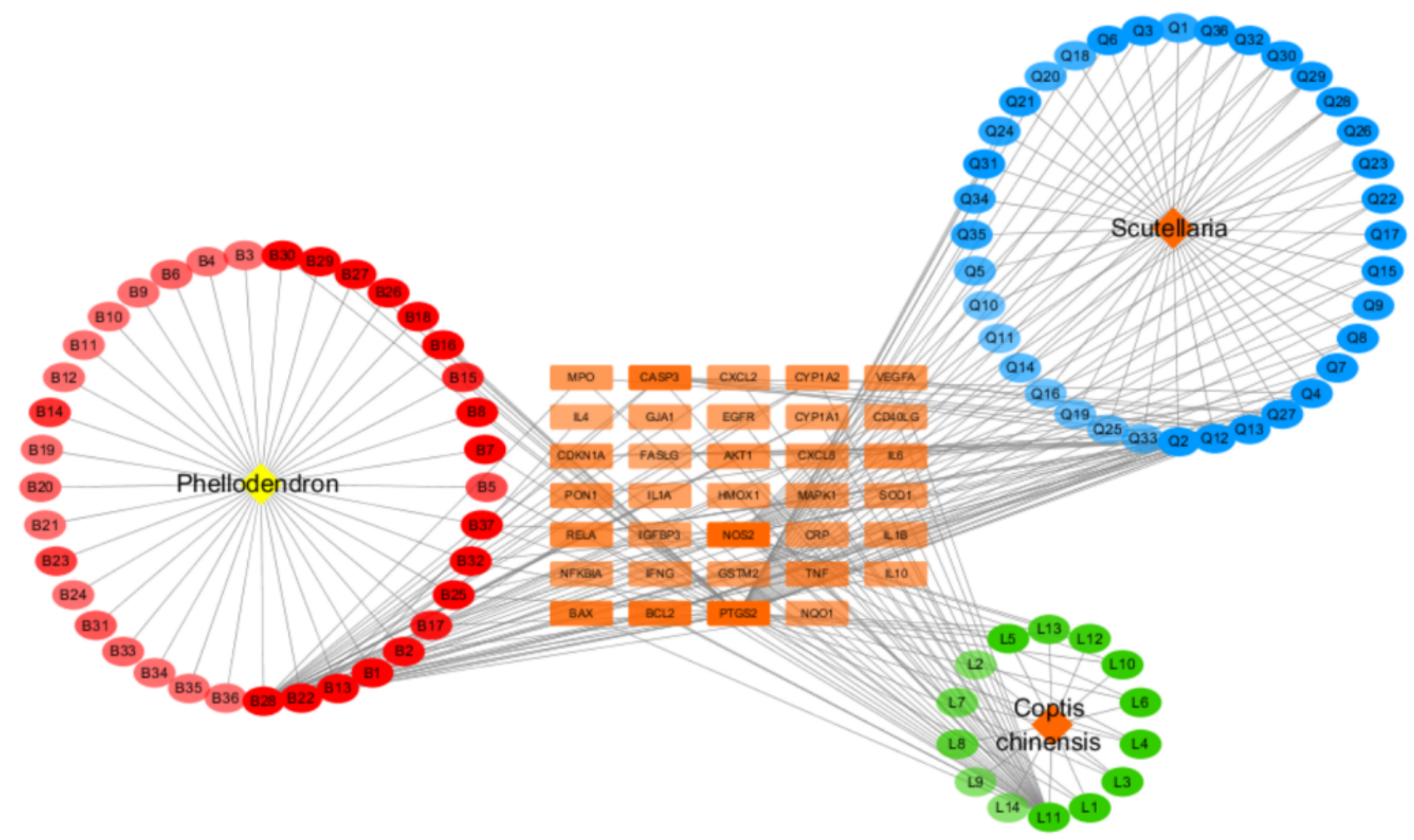

Compounds of Phellodendron

Compounds of Coptis chinensis

Compounds of Scutellaria

Chinese herbal medicine

Intersection target of $\mathrm{HHH}$ and $\mathrm{BD}$

Figure 2

The "drug-active compound-target" network of the HMI 


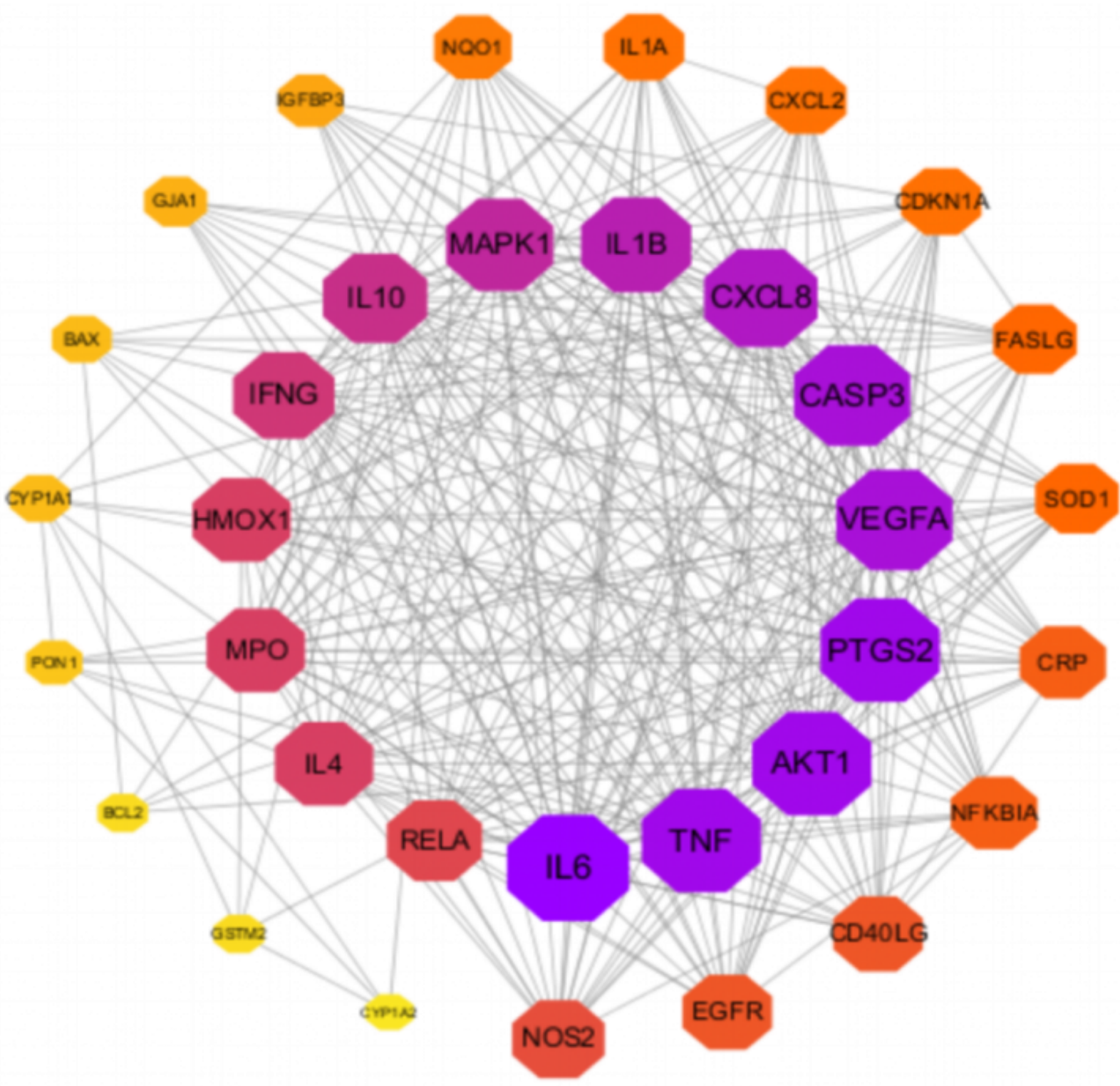

\section{Figure 3}

PPI network of the key target of $\mathrm{HRH}$ for the treatment of bacillary dysentery 


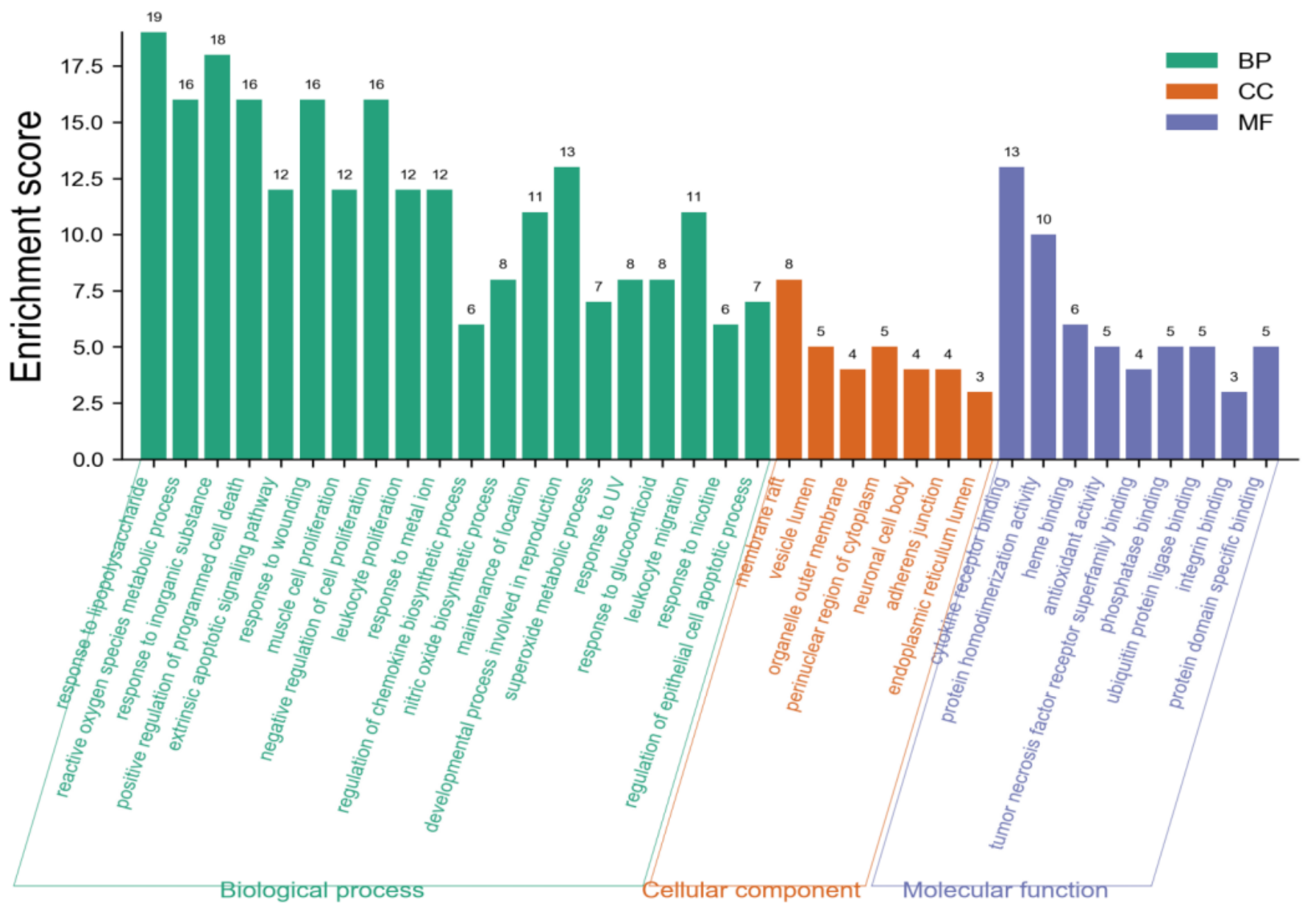

Figure 4

The GO enrichment analysts of the core target of $\mathrm{HHH}$ for the treatment of bacillary dysentery 


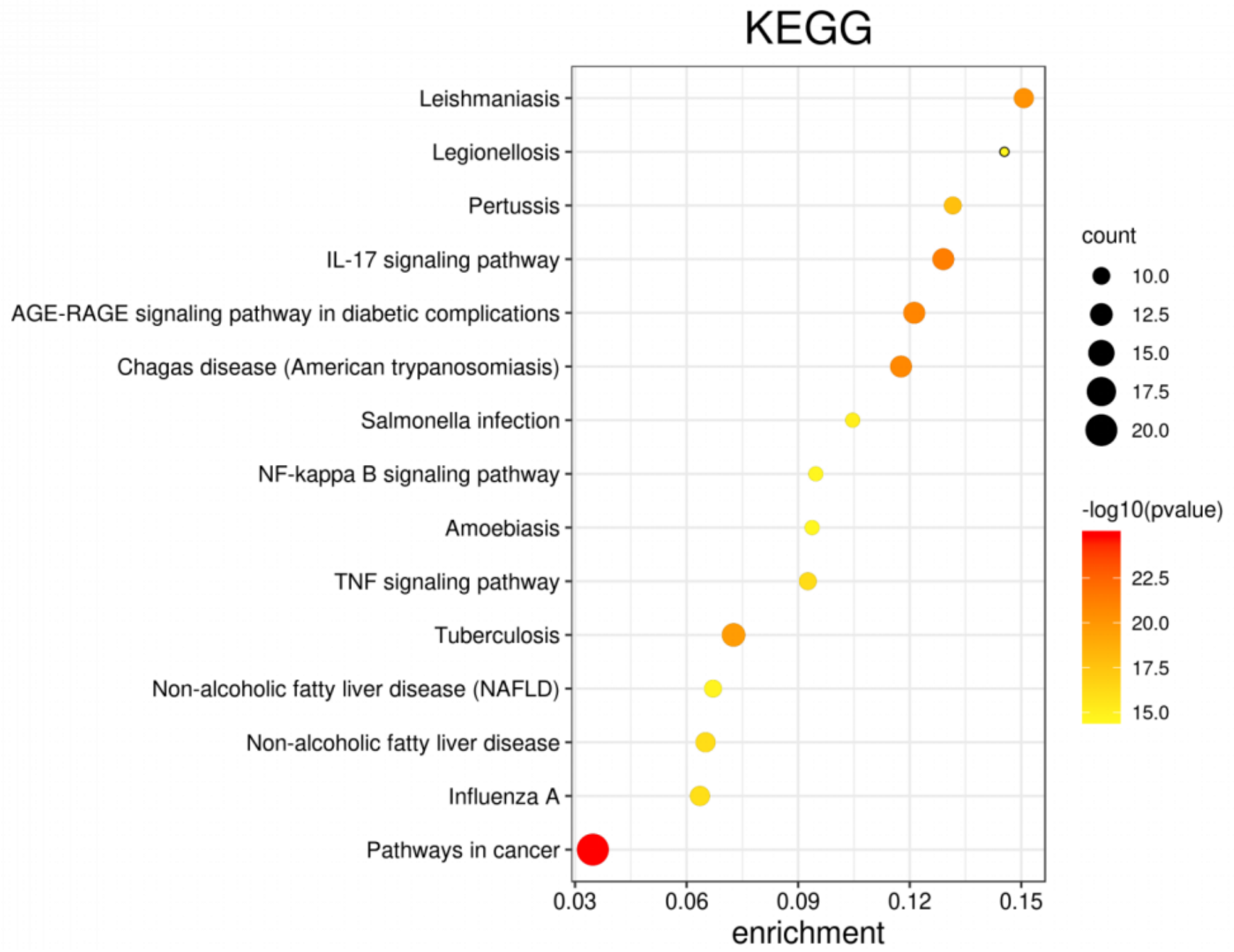

Figure 5

Enrichment analysts of KEGG , the core target of bacillary dysentery by $\mathrm{HHH}$ 


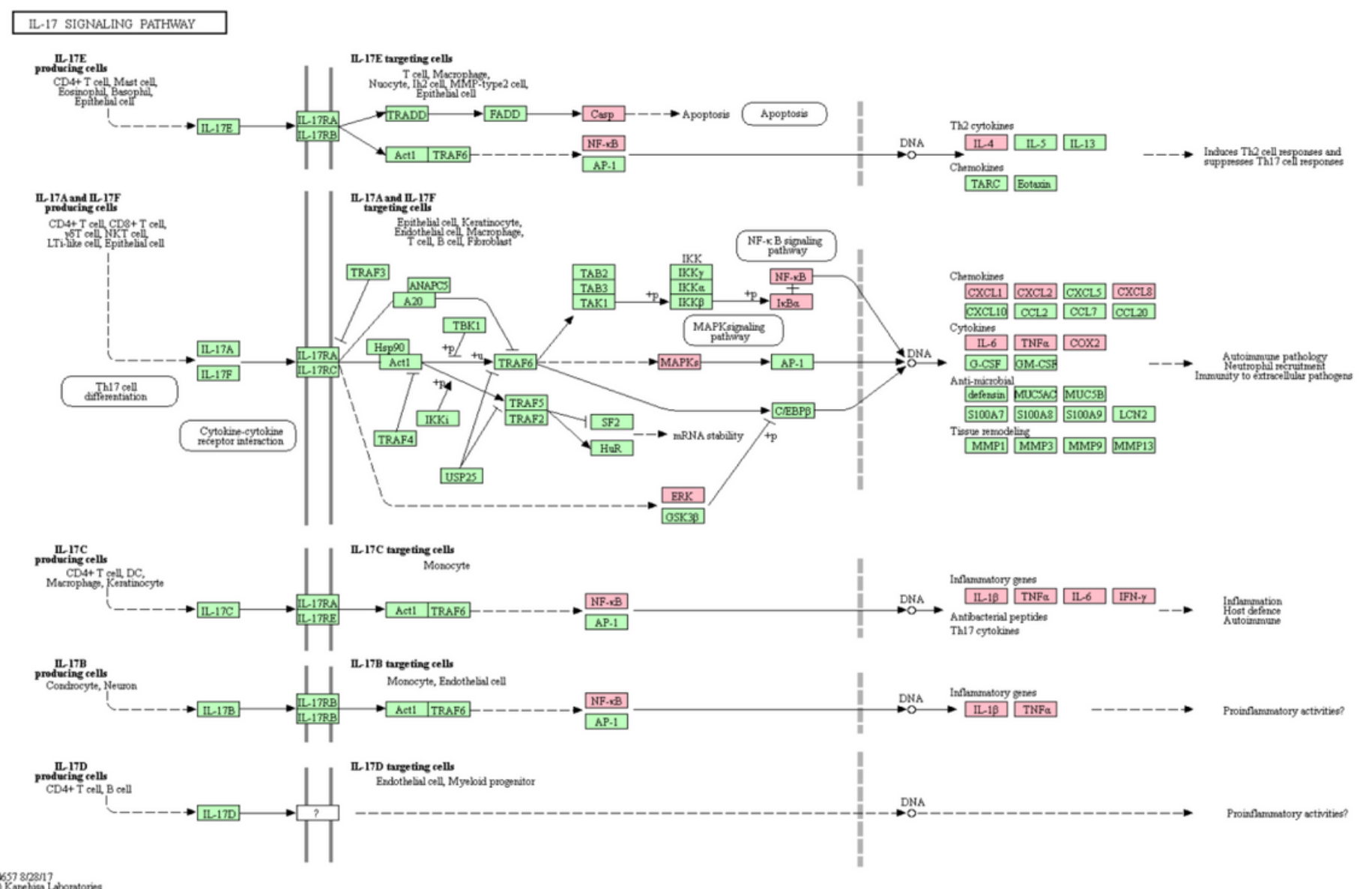

\section{Figure 6}

IL-17 signaling pathway of $\mathrm{HHH}$ for the treatment of bacillary dysentery 


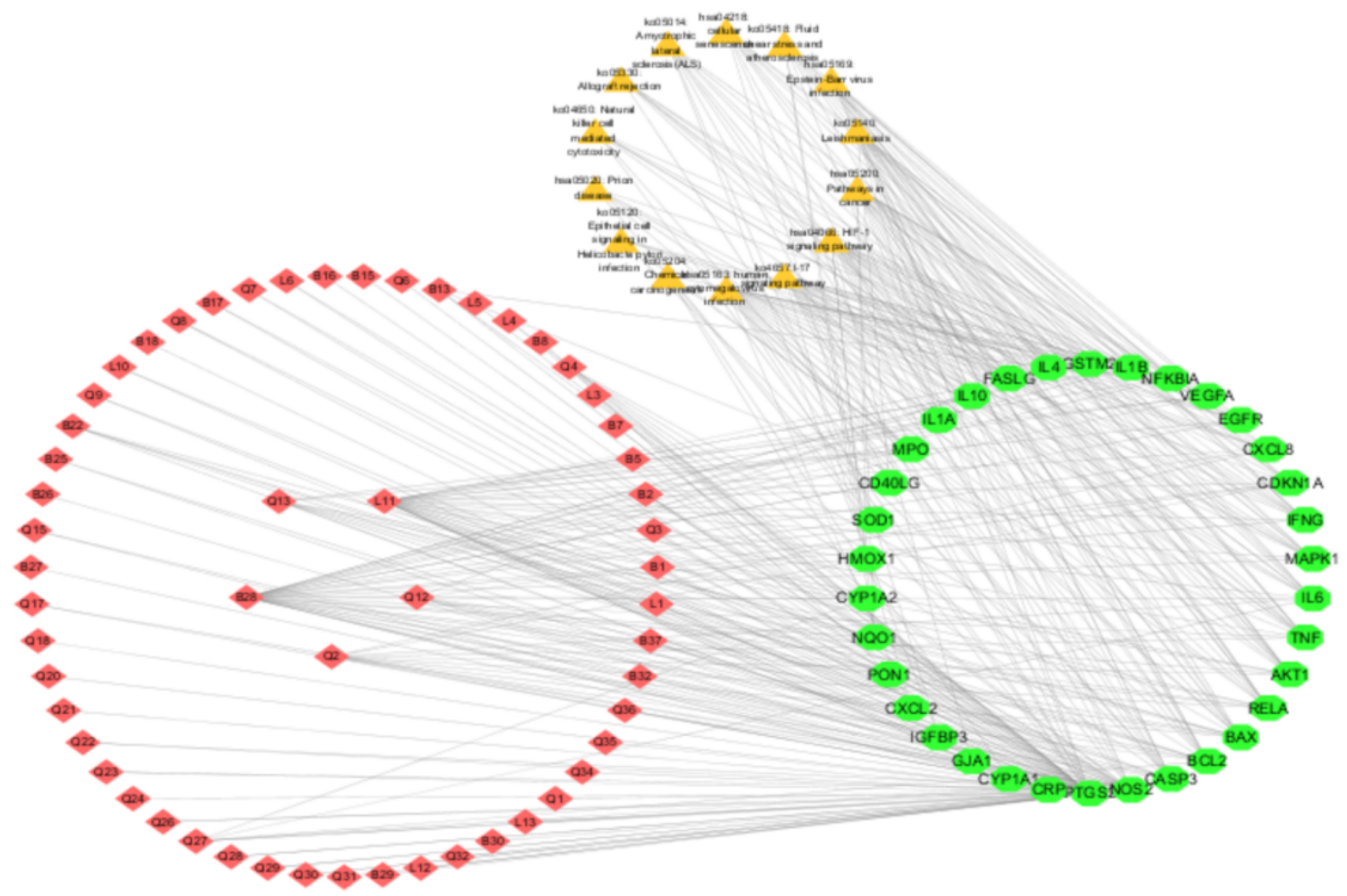

$>$ Active compound

Pathway

\section{Intersection target of $\mathrm{HHH}$ and $\mathrm{BD}$}

\section{Figure 7}

The "active compound-gene target-pathway" network of BH1-1 Result of molecular docking 

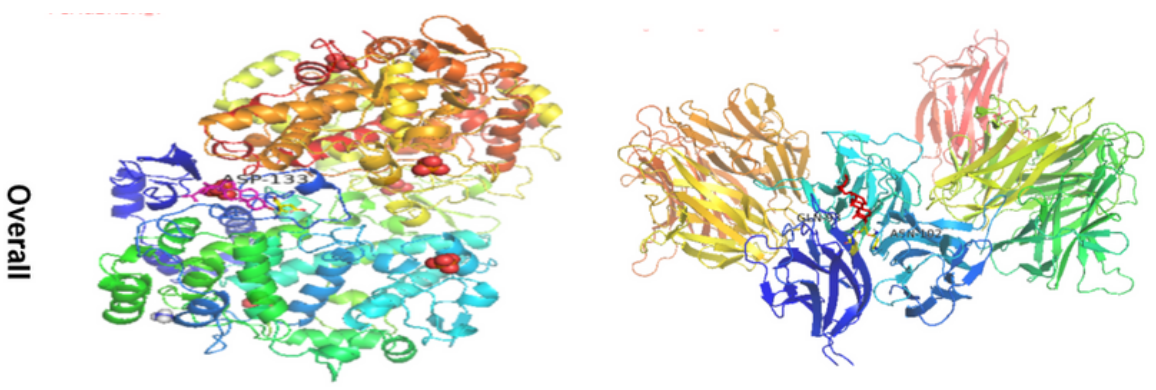

A、 beta-sitosterolPTGS2

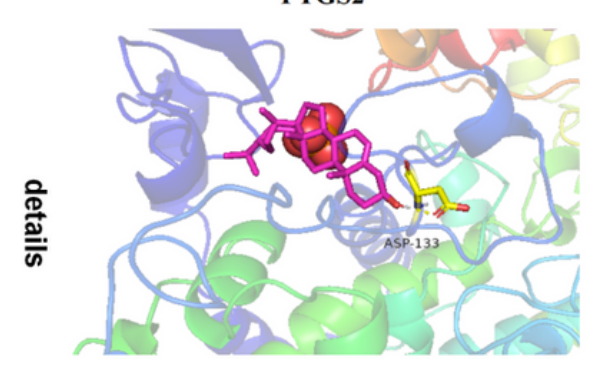

A、beta-sitosterol-
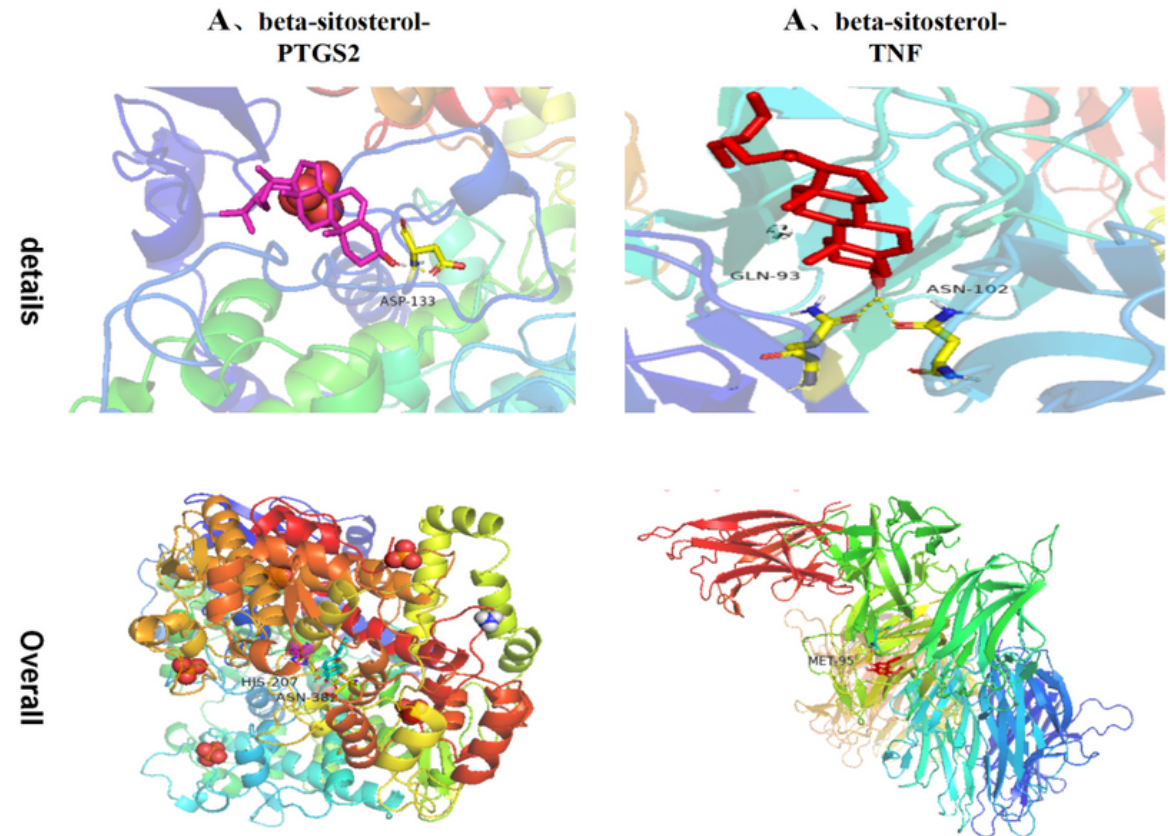

B、baicalein-PTGS2

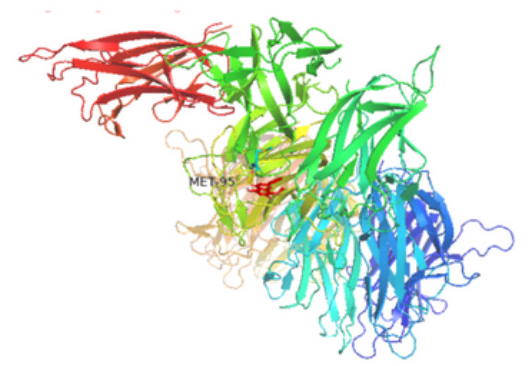

B、baicalein-TNF
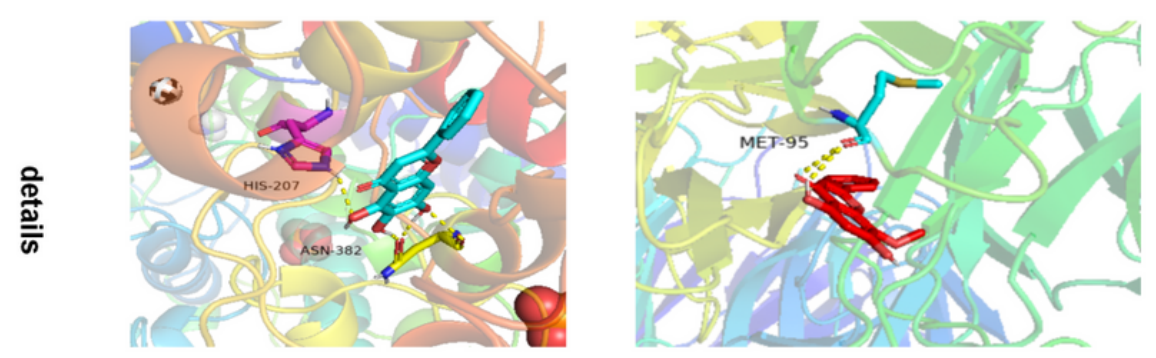

Figure 8

Core active compound (A, bea-snosterol, B, baicalein)-core target (PTOS2, TNF) molecular docking results model diagram 